\title{
Violation of Sum Rules for Twist-3 Parton Distributions in QCD
}

\author{
Matthias Burkardt ${ }^{1}$ and Yuji KolKe ${ }^{2}$ \\ ${ }^{1}$ Dept. of Physics, New Mexico State University, Las Cruces, NM 88003, USA \\ ${ }^{2}$ Dept. of Physics, Niigata University, Niigata 950-2181, Japan
}

Version of November 4, 2018

\begin{abstract}
:
Sum rules for twist-3 distributions are reexamined. Integral relations between twist-3 and twist-2 parton distributions suggest the possibility for a $\delta$-function at $x=0$. We confirm and clarify this result by constructing $h_{L}$ and $h_{L}^{3}$ (quark-gluon interaction dependent part of $h_{L}$ ) explicitly from their moments for a one-loop dressed massive quark. The physics of these results is illustrated by calculating $h_{L}\left(x, Q^{2}\right)$ using light-front time-ordered pQCD to $\mathcal{O}\left(\alpha_{S}\right)$ on a quark target. A $\delta(x)$ term is also found in $e\left(x, Q^{2}\right)$, but not in $g_{T}\left(x, Q^{2}\right)$, to this order in $\mathcal{O}\left(\alpha_{S}\right)$.
\end{abstract}




\section{Introduction}

Ongoing experiments with polarized beams and/or targets conducted at RHIC, HERMES and COMPASS etc are providing us with important information on the spin distribution carried by quarks and gluons in the nucleon. They are also enabling us to extract information on the higher twist distributions which represent the effect of quark-gluon correlations. In particular, the twist-3 distributions $g_{T}\left(x, Q^{2}\right)$ and $h_{L}\left(x, Q^{2}\right)$ are unique in that they appear as a leading contribution in some spin asymmetries: For example, $g_{T}$ can be measured in the transversely polarized lepton-nucleon deep inelastic scattering and $h_{L}$ appears in the longitudinal-transverse spin asymmetry in the polarized nucleon-nucleon Drell-Yan process [1]. The purpose of this paper is to reexamine the validity of the sum rules for these twist-3 distributions.

The complete set of the twist-3 quark distributions in our interest are given as the light-cone corelation functions in a parent hadron with momentum $P$, spin $S$ and mass $M$ :

$$
\begin{aligned}
& \int \frac{d \lambda}{2 \pi} e^{i \lambda x}\left\langle P S\left|\bar{\psi}(0) \gamma^{\mu} \gamma_{5} \psi(\lambda n)\right|_{Q^{2}} \mid P S\right\rangle \\
& =2\left[g_{1}\left(x, Q^{2}\right) p^{\mu}(S \cdot n)+g_{T}\left(x, Q^{2}\right) S_{\perp}^{\mu}+M^{2} g_{3}\left(x, Q^{2}\right) n^{\mu}(S \cdot n)\right], \\
& \int \frac{d \lambda}{2 \pi} e^{i \lambda x}\left\langle P S\left|\bar{\psi}(0) \sigma^{\mu \nu} i \gamma_{5} \psi(\lambda n)\right|_{Q^{2}} \mid P S\right\rangle=2\left[h_{1}\left(x, Q^{2}\right)\left(S_{\perp}^{\mu} p^{\nu}-S_{\perp}^{\nu} p^{\mu}\right) / M\right. \\
& \left.\quad+h_{L}\left(x, Q^{2}\right) M\left(p^{\mu} n^{\nu}-p^{\nu} n^{\mu}\right)(S \cdot n)+h_{3}\left(x, Q^{2}\right) M\left(S_{\perp}^{\mu} n^{\nu}-S_{\perp}^{\nu} n^{\mu}\right)\right], \\
& \int \frac{d \lambda}{2 \pi} e^{i \lambda x}\left\langle P S|\bar{\psi}(0) \psi(\lambda n)|_{Q^{2}} \mid P S\right\rangle=2 M e\left(x, Q^{2}\right),
\end{aligned}
$$

where two light-like vectors $p$ and $n$ are introduced by the relation $p^{2}=n^{2}=0, n^{+}=p^{-}=0$, $P^{\mu}=p^{\mu}+\frac{M^{2}}{2} n^{\mu}$ and $S^{\mu}$ is decomposed as $S^{\mu}=(S \cdot n) p^{\mu}+(S \cdot p) n^{\mu}+S_{\perp}^{\mu}$. The variable $x$ represents the parton's light-cone momentum fraction and each function has a support for $x$ on $[-1,1]$. The anti-quark distributions $\bar{g}_{1, T, 3}\left(x, Q^{2}\right), \bar{h}_{1, L, 3}\left(x, Q^{2}\right)$ are obtained by the replacement of $\psi$ into its charge conjugation field $C \bar{\psi}^{T}$ in (1.1)-(1.3) and are related to the quark distributions as $\bar{g}_{1, T, 3}\left(x, Q^{2}\right)=g_{1, T, 3}\left(-x, Q^{2}\right)$ and $\bar{h}_{1, T, 3}\left(x, Q^{2}\right)=-h_{1, T, 3}\left(-x, Q^{2}\right)$. The sum rules in our interest are obtained by taking the first moment of the above relations. For example, from (1.1), one obtains

$$
\begin{aligned}
& \left\langle P S\left|\bar{\psi}(0) \gamma^{\mu} \gamma_{5} \psi(0)\right|_{Q^{2}} \mid P S\right\rangle \\
& \quad=2 \int_{-1}^{1} d x\left[g_{1}\left(x, Q^{2}\right) p^{\mu}(S \cdot n)+g_{T}\left(x, Q^{2}\right) S_{\perp}^{\mu}+M^{2} g_{3}\left(x, Q^{2}\right) n^{\mu}(S \cdot n)\right] .
\end{aligned}
$$

From rotational invariance, it follows that the left hand side of (1.4) is proportional to the spin vector $S^{\mu}$ and thus $g_{1, T, 3}\left(x, Q^{2}\right)$ must satisfy

$$
\int_{-1}^{1} d x g_{1}\left(x, Q^{2}\right)=\int_{-1}^{1} d x g_{T}\left(x, Q^{2}\right),
$$




$$
\int_{-1}^{1} d x g_{1}\left(x, Q^{2}\right)=2 \int_{-1}^{1} d x g_{3}\left(x, Q^{2}\right) .
$$

The same argument for (1.2) leads to the sum rule relations for $h_{1, L, 3}\left(x, Q^{2}\right)$ :

$$
\begin{aligned}
\int_{-1}^{1} d x h_{1}\left(x, Q^{2}\right) & =\int_{-1}^{1} d x h_{L}\left(x, Q^{2}\right), \\
\int_{-1}^{1} d x h_{1}\left(x, Q^{2}\right) & =2 \int_{-1}^{1} d x h_{3}\left(x, Q^{2}\right) .
\end{aligned}
$$

The sum rule (1.5) is known as Burkhardt-Cottingham sum rule [2] and (1.7) was first derived in Refs. [3, 4, 5]. Since the twist-4 distributions $g_{3}, h_{3}$ are unlikely to be measured experimentally, the sum rules involving those functions (1.6) and (1.8) are practically useless and will not be addressed in the subsequent discussions. Since the left hand side of (1.5) and (1.7) are, respectively, the axial charge and the tensor charge, the integral itself in the right hand side of (1.5)-(1.8) are finite. As is clear from the above derivation, these sum rules are mere consequences of the rotational invariance and there is no doubt in its validity in mathematical sense. However, if one try to confirm those sum rules by experiment, a great care is required to perform the integral including $x=0$. For example, in DIS, $x$ is identified as the Bjorken's variable $x_{B}=Q^{2} / 2 P \cdot q$ and $x=0$ corresponds to $P \cdot q \rightarrow \infty$ and this limit can never be achieved in the rigorous sense. Accordingly, if $h_{L}\left(x, Q^{2}\right)$ has a contribution proportional to $\delta(x)$ and $h_{1}\left(x, Q^{2}\right)$ does not, experimental measurement can never confirm the sum rule (1.7) but would rather claim the violation of the sum rule. Such a contribution has already been suggested in Refs. [3, 5]. In Refs. [6, 7, 8] it was also argued that the integral of $h_{L}\left(x, Q^{2}\right)-h_{1}\left(x, Q^{2}\right)$ can be related to the value of certain 'surface terms' that appear in formal manipulations involving integrations by parts.

The purpose of this paper is to reexamine the sum rules involving the first moment of the twist-3 distributions. In particular we will argue that the twist-3 distribution $h_{L}\left(x, Q^{2}\right)$ has a potential $\delta$-singularity at $x=0$, assuming that the twist-2 distributions $g_{1}\left(x, Q^{2}\right)$ and $h_{1}\left(x, Q^{2}\right)$ do not have such singularity. The paper is organized as follows: In section 2 , we examine the sum rule for $h_{L}$. Starting from the general decomposition of $h_{L}$ based on the QCD equation of motion, we will show that it contains a function $h_{L}^{m}$ which has $\delta(x)$-singularity (Section 2.1). In Section 2.2, we construct $h_{L}$ for a massive quark ¿from the moment of $h_{L}^{3}$ in the one-loop level and show that the $h_{L}^{3}$ also has an $\delta(x)$-singularity, which together with the singularity in $h_{L}^{m}$ will give rise to a $\delta(x)$ singularity in $h_{L}$ itself. In section 2.3, we will perform an explicit light-cone calculation of $h_{L}$ in the one-loop level to confirm the result in the previous sections. Sections 3 and 4 are, respectively, devoted to similar examination for the sum rules for $e\left(x, Q^{2}\right)$ and $g_{T}\left(x, Q^{2}\right)$.

\section{$2 h_{L}\left(x, Q^{2}\right)$ for a massive quark}




\section{$2.1 \delta(x)$-functions in $h_{L}\left(x, Q^{2}\right)$}

The OPE analysis of the correlation function (1.2) allows us to decompose $h_{L}\left(x, Q^{2}\right)$ into the contribution expressed in terms of twist-2 distributions and the rest which we call $h_{L}^{3}\left(x, Q^{2}\right)$. Since the scale dependence of each distribution is inessential in the following discussion, we shall omit it in this subsection for simplicity. Introducing the notation for the moments on $[-1,1]$

$$
\mathcal{M}_{n}\left[h_{L}\right] \equiv \int_{-1}^{1} d x x^{n} h_{L}(x)
$$

this decomposition is given in terms of the moment relation [1]]:

$$
\begin{aligned}
& \mathcal{M}_{n}\left[h_{L}\right]=\frac{2}{n+2} \mathcal{M}_{n}\left[h_{1}\right]+\frac{n}{n+2} \frac{m_{q}}{M} \mathcal{M}_{n-1}\left[g_{1}\right]+\mathcal{M}_{n}\left[h_{L}^{3}\right], \quad(n \geq 1) \\
& \mathcal{M}_{0}\left[h_{L}\right]=\mathcal{M}_{0}\left[h_{1}\right]
\end{aligned}
$$

with the condition

$$
\begin{aligned}
& \mathcal{M}_{0}\left[h_{L}^{3}\right]=0 \\
& \mathcal{M}_{1}\left[h_{L}^{3}\right]=0
\end{aligned}
$$

By inverting the moment relation, one finds

$$
\begin{aligned}
h_{L}(x)= & h_{L}^{W W}(x)+h_{L}^{m}(x)+h_{L}^{3}(x) \\
= & \left\{\begin{array}{c}
2 x \int_{x}^{1} d y \frac{h_{1}(y)}{y^{2}}+\frac{m_{q}}{M}\left[\frac{g_{1}(x)}{x}-2 x \int_{x}^{1} d y \frac{g_{1}(y)}{y^{3}}\right]+h_{L}^{3}(x) \\
-2 x \int_{-1}^{x} d y \frac{h_{1}(y)}{y^{2}}+\frac{m_{q}}{M}\left[\frac{g_{1}(x)}{x}+2 x \int_{-1}^{x} d y \frac{g_{1}(y)}{y^{3}}\right]+h_{L}^{3}(x), \\
(x<0)
\end{array}\right.
\end{aligned}
$$

where the first and second terms in Eq. (2.6) denote the corresponding terms in (2.7). In this notation the sum rule (2.3) and the condition (2.4) implies Đ

$$
\mathcal{M}_{0}\left[h_{L}^{m}\right]=0
$$

If one naively integrates (2.7) over $x$ at $x>0$ and $x<0$, while dropping all surface terms one arrives at $\int_{0}^{1} d x h_{L}(x)=\int_{0}^{1} d x h_{1}(x)+\int_{0}^{1} d x h_{L}^{3}(x)$ and likewise for $\int_{-1}^{0} d x h_{L}(x)$. This

\footnotetext{
${ }^{1}$ More precisely, the original OPE tells us $\mathcal{M}_{0}\left[h_{L}^{3}+h_{L}^{m}\right]=0$. But as long as $g_{1}\left(0_{ \pm}\right)$is finite, which we will assume, this is equivalent to stronger relations (2.4) and (2.8).
} 
result, in combination with (2.4), implies that $\int_{-1}^{1} d x h_{L}=\int_{-1}^{1} d x h_{1}$. In the following we will argue that this procedure may be wrong due to the potentially very singular behavior of the functions involved near $x=0$. Investigating this issue in detail will be the main purpose of this paper.

We first address the potential singularity at $x=0$ in the integral expression for $h_{L}^{m}(x)$ in (2.7). In order to regulate the region near $x=0$, we first multiply $h_{L}^{m}(x)$ by $x^{\beta}$, integrate from 0 to 1 and let $\beta \rightarrow 0$. This yields

$$
\int_{0+}^{1} d x h_{L}^{m}(x)=\frac{m_{q}}{2 M} \lim _{\beta \rightarrow 0} \beta \int_{0}^{1} d x x^{\beta-1} g_{1}(y)=\frac{m_{q}}{2 M} g_{1}(0+),
$$

while multiplying Eq. (2.7) by $|x|^{\beta}$ and integration from -1 to 0 yields

$$
\int_{-1}^{0-} d x h_{L}^{m}(x)=-\frac{m_{q}}{2 M} \lim _{\beta \rightarrow 0} \beta \int_{-1}^{0-} d x|x|^{\beta-1} g_{1}(y)=-\frac{m_{q}}{2 M} g_{1}(0-),
$$

where we have assumed that $g_{1}(0+)$ and $g_{1}(0-)$ are finite. Adding these results we have

$$
\int_{-1}^{0-} d x h_{L}^{m}(x)+\int_{0+}^{1} d x h_{L}^{m}(x)=\frac{m_{q}}{2 M}\left(g_{1}(0+)-g_{1}(0-)\right) .
$$

Since we have the sum rule (2.8) and, in general, $\lim _{x \rightarrow 0} g_{1}(x)-g_{1}(-x) \neq 0$, I we are lead to conclude

$$
h_{L}^{m}(x)=h_{L}^{m}(x)_{r e g}-\frac{m_{q}}{2 M}\left(g_{1}(0+)-g_{1}(0-)\right) \delta(x),
$$

where $h_{L}^{m}(x)_{\text {reg }}$ stands for the part which is defined by the integral in (2.7) at $x>0$ and $x<0$ and is regular at $x=0$. Since it is unlikely that $h_{L}^{W W}(x)$ contains a $\delta$-function at the origin, the relation (2.12) indicates that $h_{L}$ has a $\delta(x)$ term unless $h_{L}^{3}(x)$ has a $\delta(x)$ term and it cancels the above singularity in $h_{L}^{m}(x)$.

Equation (2.12) clearly demonstrate that the functions constituting $h_{L}(x)$ are more singular near $x=0$ than previously assumed and that great care needs to be taken when replacing integrals over nonzero values of $x$ by integrals that involve the origin. In particular, if it turns out that $h_{L}(x)$ itself contains a $\delta(x)$ term, then (2.3) implies

$$
\int_{0+}^{1}\left(h_{L}(x)-h_{1}(x)\right)+\int_{-1}^{0-}\left(h_{L}(x)-h_{1}(x)\right) \neq 0,
$$

since $h_{1}(x)$ is free from singularity at $x=0$ :

$$
\int_{-1}^{0-} d x h_{1}(x)+\int_{0+}^{1} d x h_{1}(x)=\int_{-1}^{1} d x h_{1}(x) .
$$

\footnotetext{
${ }^{2}$ For example, dressing a quark perturbatively at $\mathcal{O}\left(\alpha_{S}\right)$ yields $g_{1}(0+) \neq 0$ and $g_{1}(0-) \equiv \bar{g}_{1}(0+)=0$.
} 
Accordingly an attempt to verify the " $h_{L}$-sum rule" [3] would obviously fail.

However, as we mentioned earlier, in order to see whether the $\delta(x)$ identified in (2.12) eventually survives or not in $h_{L}(x)$, we have to investigate the behavior of $h_{L}^{3}(x)$ at $x=0$. To this end we will explicitly construct $h_{L}(x)$ for a massive quark to $\mathcal{O}\left(\alpha_{S}\right)$.

\section{2 $h_{L}\left(x, Q^{2}\right)$ from the moment relations}

In this subsection we will construct $h_{L}\left(x, Q^{2}\right)$ for a massive quark to $\mathcal{O}\left(\alpha_{S}\right)$ from the one-loop calculation of $\mathcal{M}_{n}\left[h_{L}^{3}\right]$.

One-loop calculation for a massive quark (mass $m_{q}$ ) gives $h_{L}\left(x, Q^{2}\right)$ in the following form:

$$
h_{L}\left(x, Q^{2}\right)=h_{L}^{(0)}(x)+\frac{\alpha_{S}}{2 \pi} C_{F} \ln \frac{Q^{2}}{m_{q}^{2}} h_{L}^{(1)}(x),
$$

where the scale $Q^{2}$ is introduced as an ultraviolet cutoff and the $C_{F}=4 / 3$ is the color factor. $h_{L}^{W W, 3, m(0,1)}(x)$ are defined similarly. $g_{1}^{(0)}(x)=h_{1}^{(0)}(x)=\delta(1-x)$ gives $h_{L}^{(0)}(x)=\delta(1-x)$, as it should. One loop calculation for $g_{1}(x)$ and $h_{1}(x)$ for a quark yields the well known splitting functions [9, 10]:

$$
\begin{aligned}
& g_{1}^{(1)}(x)=\frac{1+x^{2}}{[1-x]_{+}}+\frac{3}{2} \delta(1-x), \\
& h_{1}^{(1)}(x)=\frac{2 x}{[1-x]_{+}}+\frac{3}{2} \delta(1-x) .
\end{aligned}
$$

Inserting these equations into the defining equation in (2.7), one obtains

$$
\begin{aligned}
h_{L}^{W W(1)}(x) & =3 x+4 x \ln \frac{1-x}{x} \\
h_{L}^{m(1)}(x) & =\frac{2}{(1-x)_{+}}-4 x \ln \frac{1-x}{x}-3+\frac{3}{2} \delta(1-x)+\left(3 x-\frac{3}{2} \delta(1-x)\right)-\frac{1}{2} \delta(x) \\
& =\frac{2}{(1-x)_{+}}-4 x \ln \frac{1-x}{x}-3+3 x-\frac{1}{2} \delta(x) .
\end{aligned}
$$

In the first line of (2.19), the term $\left(3 x-\frac{3}{2} \delta(1-x)\right)$ comes from the self-energy correction, i.e. from expanding $M=m_{q}\left[1+\frac{\alpha_{S}}{2 \pi} C_{F} \frac{3}{2} \ln \frac{Q^{2}}{m_{q}^{2}}\right]$ in Eq. (2.6), and $-\frac{1}{2} \delta(x)=-\frac{1}{2} g_{1}(0+) \delta(x)$ in $h_{L}^{m(1)}(x)$ accounts for the second term on the righthand side of Eq. (2.12). This term is necessary to reproduce the original moment relation (2.8). We also note that $h_{L}^{W W(1)}$ does not have any singularity at $x=0$ and satisfies $\int_{0}^{1} d x h_{L}^{W W(1)}(x)=\int_{0}^{1} d x h_{1}^{(1)}(x)$ as it should. 
$h_{L}^{(1)}(x)$ can be constructed if we know the purely twist-3 part $h_{L}^{3(1)}(x)$ in the one-loop level. $h_{L}^{3}\left(x, \mu^{2}\right)$ can be written in terms of the quark-gluon light-cone correlation function as $\left(\xi^{2}=0, \xi^{+}=0\right) 11$, 12, 13,

$$
\begin{aligned}
h_{L}^{3}\left(x, Q^{2}\right)= & \frac{i P^{+}}{M} \int_{-\infty}^{\infty} \frac{d \xi^{-}}{2 \pi} e^{2 i x P^{+} \xi^{-}} \int_{0}^{1} u d u \int_{-u}^{u} t d t \\
& \times\left\langle P S_{\|}\left|\bar{\psi}\left(-u \xi^{-}\right) i \gamma_{5} \sigma_{\mu \alpha} g F_{\nu}^{\alpha}\left(t \xi^{-}\right) \xi^{\mu} \xi^{\nu} \psi\left(u \xi^{-}\right)\right|_{Q^{2}} \mid P S_{\|}\right\rangle .
\end{aligned}
$$

Starting from this expression, one can, in principle, obtain $h_{L}^{3(1)}(x)$. Alternatively, and this is the approach that we will use, one can construct it from already existing one-loop calculation of its moments. Taking the $n$-th moment of (2.20) or from the OPE analysis of $h_{L}$ [1], we have

$$
\int_{-1}^{1} d x x^{n} h_{L}^{3}\left(x, Q^{2}\right)=\sum_{k=2}^{[(n+1) / 2]}\left(1-\frac{2 k}{n+2}\right) \frac{1}{2 M}\left\langle P S_{\|}\left|R_{n, k}\left(Q^{2}\right)\right| P S_{\|}\right\rangle
$$

with

$$
R_{n, k}\left(Q^{2}\right)=\left.\frac{1}{2} \bar{\psi}(0) \sigma^{\alpha \beta} n_{\beta} i \gamma_{5}(i n \cdot D)^{n-k} i g F_{\alpha}^{\nu} n_{\nu}(i n \cdot D)^{k-2} \psi(0)\right|_{Q^{2}}-(k \rightarrow n-k+2)(2
$$

One-loop renormalization of $h_{L}$ was completed in [14] and the mixing matrix for the local operators contributing to the moments of $h_{L}\left(x, Q^{2}\right)$ was presented. In particular, matrix elements of $R_{n, k}$ for a massive quark is given in eq.(3.18) of [14. Since $h_{L}^{(1)}(x)$ for the anti-quark is zero, we obtain for the moment of the quark distribution as

$$
\begin{aligned}
\int_{0}^{1} d x x^{n} h_{L}^{3(1)}(x) & =2 \sum_{l=2}^{[(n+1) / 2]}\left(1-\frac{2 l}{n+2}\right)\left(\frac{1}{[l-1]_{3}}-\frac{1}{[n-l+1]_{3}}\right) \\
& =\frac{3}{n+1}-\frac{6}{n+2}+\frac{1}{2}
\end{aligned}
$$

for $n \geq 2$ with $[k]_{3} \equiv k(k+1)(k+2)$. [The prefactor in $(2.23)$ is determined by comparison with the anomalous dimension for $h_{1}$ and by noting that the operator basis for the quark mass operator in [14] has a sign opposite to those for $g_{1}$.] From this result and the defining relation for the lowest two moments of $h_{L}^{3}$, (2.4) and (2.5), we can construct $h_{L}^{3(1)}(x)$ as

$$
h_{L}^{3(1)}(x)=3-6 x+\frac{1}{2} \delta(1-x)-\frac{1}{2} \delta(x)
$$

We emphasize that the $-1 / 2 \delta(x)$ in (2.24) is necessary to reproduce the $n=0$ moment of $h_{L}^{3(1)}(x)$. From (2.18), (2.19) and (2.24), one obtains

$$
h_{L}\left(x, Q^{2}\right)=\delta(1-x)+\frac{\alpha_{S}}{2 \pi} \ln \frac{Q^{2}}{m_{q}^{2}} C_{F}\left[\frac{2}{[1-x]_{+}}+\frac{1}{2} \delta(1-x)-\delta(x)\right] \text {. }
$$


We remark that the above calculation indicates that the $\delta(x)$ term appears not only in $h_{L}^{m}$ but also in $h_{L}^{3}$. Furthermore they do not cancel but add up to give rise to $-\delta(x)$ in $h_{L}\left(x, Q^{2}\right)$ itself.

In the next subsection we will confirm Eq. (2.25) through a direct calculation of $h_{L}\left(x, Q^{2}\right)$ for a quark.

\subsection{Light-cone calculation of $h_{L}\left(x, Q^{2}\right)$}

In order to illustrate the physical origin of the $\delta(x)$ terms in $h_{L}(x)$, and in order to develop a more general and convenient procedure for calculating such terms, we shall now evaluate $h_{L}(x)$ using time-ordered light-front (LF) perturbation theory. The general method has been outlined in Ref. [16] and we will restrict ourselves here to the essential steps only.

There are two equivalent ways to perform time-ordered LF perturbation theory: one can either work with the LF Hamiltonian for QCD and perform old-fashioned perturbation theory (the method employed in Ref. [16]), or one can start from Feynman perturbation theory and integrate over the LF-energy $k^{-}$first. We found the second approach more convenient for the one-loop calculation of $h_{L}(x)$ and this is what we will use in the following.

In LF gauge, $A^{+}=0$, parton distributions can be expressed in terms of LF momentum densities $\left(k^{+}\right.$-densities). Therefore, one finds for a parton distribution, characterized by the Dirac matrix $\Gamma$ at $\mathcal{O}\left(\alpha_{S}\right)$ and for $0<x<1$

$f_{\Gamma}(x) \bar{u}(p) \Gamma u(p)=-i g^{2} \bar{u}(p) \int \frac{d^{4} k}{(2 \pi)^{4}} \delta\left(x-\frac{k^{+}}{p^{+}}\right) \gamma^{\mu} \frac{1}{\not k-m_{q}+i \varepsilon} \Gamma \frac{1}{\not k-m_{q}+i \varepsilon} \gamma^{\nu} u(p) D_{\mu \nu}(p-k)$,

where

$$
D_{\mu \nu}(q)=\frac{1}{q^{2}+i \varepsilon}\left[g_{\mu \nu}-\frac{q_{\mu} n_{\nu}+n_{\mu} q_{\nu}}{q n}\right]
$$

is the gauge field propagator in LF gauge, and $n^{\mu}$ is a light-like vector such that $n A=A^{+} \sim$ $\left(A^{0}+A^{3}\right) / \sqrt{2}$ for any four vector $A^{\mu}$.

The $k^{-}$integrals in expressions like Eq. (2.26) are performed using Cauchy's theorem, yielding for $0<k^{+}<p^{+}$

$$
\begin{aligned}
-i \int \frac{d k^{-}}{2 \pi} \frac{1}{\left(k^{2}-m_{q}^{2}+i \varepsilon\right)^{2}} \frac{1}{(p-k)^{2}+i \varepsilon}= & \frac{1}{\left(2 k^{+}\right)^{2}} \frac{1}{2\left(p^{+}-k^{+}\right)} \frac{1}{\left(p^{-}-\frac{m_{q}^{2}+\mathbf{k}_{\perp}^{2}}{2 k^{+}}-\frac{\left(\mathbf{p}_{\perp}-\mathbf{k}_{\perp}\right)^{2}}{2\left(p^{+}-k^{+}\right)}\right)^{2}} \\
& \stackrel{\mathbf{k}_{\perp \rightarrow \infty}}{\longrightarrow} \frac{1}{2 p^{+}} \frac{1-x}{\mathbf{k}_{\perp}^{4}}
\end{aligned}
$$

where we used $k^{+}=x p^{+}$. In order to integrate all terms in Eq. (2.26) over $k^{-}$, Cauchy's theorem is used to replace any factors of $k^{-}$in the numerator of Eq. (2.26) containing $k^{-}$ 
by their on-shell value at the pole of the gluon propagator

$$
k^{-} \longrightarrow \tilde{k}^{-} \equiv p^{-}-\frac{\left(\mathbf{p}_{\perp}-\mathbf{k}_{\perp}\right)^{2}}{2\left(p^{+}-k^{+}\right)}
$$

In the following we will focus on the UV divergent contributions to the parton distribution only. This helps to keep the necessary algebra at a reasonable level because we restrict ourselves to the leading behavior in $\mathbf{k}_{\perp}$, which arises from those terms in the numerator of Eq.(2.26) that are quadratic in $\mathbf{k}_{\perp}$, and therefore give rise to a logarithmically divergent $\mathbf{k}_{\perp}$ integral. The transverse momentum cutoff can be replaced by $Q^{2}$ in these expressions. The rest of the calculation is just tedious algebra and we omit the intermediate steps here.

We find for $0<x<1$ to $\mathcal{O}\left(\alpha_{S}\right)$

$$
h_{L}\left(x, Q^{2}\right)=\frac{\alpha_{S}}{2 \pi} C_{F} \ln \frac{Q^{2}}{m_{q}^{2}} \frac{2}{[1-x]_{+}},
$$

where the usual +-prescription for $\frac{1}{[1-x]_{+}}$applies at $x=1$, i.e. $\frac{1}{[1-x]_{+}}=\frac{1}{1-x}$ for $x<1$ and $\int_{0}^{1} d x \frac{1}{[1-x]_{+}}=0$. Furthermore, $h_{L}(x)=0$ for $x<0$, since anti-quarks do not occur in the $\mathcal{O}\left(\alpha_{S}\right)$ dressing of a quark. In addition to Eq. (2.30), there is also an explicit $\delta(x-1)$ contribution at $x=1$. These are familiar from twist-2 distributions, where they reflect the fact that the probability to find the quark as a bare quark is less than one due to the dressing with gluons. For higher-twist distributions, the wave function renormalization contributes is $\frac{\alpha_{S}}{2 \pi} C_{F} \ln \frac{Q^{2}}{m_{q}^{2}} \frac{3}{2} \delta(x-1)$. The same wave function renormalization also contributes at twist-3. However, for all higher twist distributions there is an additional source for $\delta(x-1)$ terms which has, in parton language, more the appearance of a vertex correction, but which arises in fact from the gauge-piece of self-energies connected to the vertex by an instantaneous fermion propagator' $\frac{\gamma^{+}}{2 p^{+}}$. For $g_{T}\left(x, Q^{2}\right)$ these have been calculated in Ref. [16] where they give an additional contribution $-\frac{\alpha_{S}}{2 \pi} C_{F} \ln \frac{Q^{2}}{m_{q}^{2}} \delta(x-1)$, i.e. the total contribution at $x=1$ for $g_{T}\left(x, Q^{2}\right)$ was found to be $\frac{\alpha_{S}}{2 \pi} C_{F} \ln \frac{Q^{2}}{m_{q}^{2}} \frac{1}{2} \delta(x-1)$. We found the same $\delta(x-1)$ terms also for $h_{L}\left(x, Q^{2}\right)$. Pl Combining the $\delta(x-1)$ piece with Eq. (2.30) we thus find

$$
h_{L}\left(x, Q^{2}\right)=\delta(x-1)+\frac{\alpha_{S}}{2 \pi} C_{F} \ln \frac{Q^{2}}{m_{q}^{2}}\left[\frac{2}{[1-x]_{+}}+\frac{1}{2} \delta(x-1)\right] \quad \text { for } \quad x>0 .
$$

Comparing this result with the well known result for $h_{1}$ [10

$$
h_{1}\left(x, Q^{2}\right)=\delta(x-1)+\frac{\alpha_{S}}{2 \pi} \ln \frac{Q^{2}}{m_{q}^{2}} C_{F}\left[\frac{2 x}{[1-x]_{+}}+\frac{3}{2} \delta(x-1)\right],
$$

\footnotetext{
${ }^{3}$ Physically, the reason why the wave function renormalization contribution depends on the twist is that in LF gauge, different components of the fermion field aquire different wave function renormalization. However, since all twist-3 parton distributions involve one LC-good and one LC-bad component, it is natural to find the same wave function renormalization for all three twist-3 distributions.
} 
one realizes that

$$
\lim _{\varepsilon \rightarrow 0} \int_{\varepsilon}^{1} d x\left[h_{L}\left(x, Q^{2}\right)-h_{1}\left(x, Q^{2}\right]=\frac{\alpha_{S}}{2 \pi} \ln \frac{Q^{2}}{m_{q}^{2}} C_{F} \neq 0,\right.
$$

i.e. if one excludes the possibly problematic region $x=0$, then the $h_{L}$-sum rule [3] is violated already for a quark dressed with gluons at order $\mathcal{O}\left(\alpha_{S}\right)$.

In the above calculation, we carefully avoided the point $x=0$. For most values of $k^{+}$, the denominator in Eq. (2.26) contains three powers of $k^{-}$when $k^{-} \rightarrow \infty$. However, when $k^{+}=0, k^{2}-m_{q}^{2}$ becomes independent of $k^{-}$and the denominator in Eq. (2.26) contains only one power of $k^{-}$. Therefore, for those terms in the numerator which are linear in $k^{-}$, f the $k^{-}$-integral diverges linearly. Although this happens only for a point of measure zero (namely at $k^{+}=0$ ), a linear divergence is indicative of a singularity of $h_{L}\left(x, Q^{2}\right)$ at that point. f To investigate the $k^{+} \approx 0$ singularity in these terms further, we consider

$$
\begin{aligned}
f\left(k^{+}, \mathbf{k}_{\perp}\right) & \equiv \int d k^{-} \frac{k^{-}}{\left(k^{2}-m_{q}^{2}+i \varepsilon\right)^{2}} \frac{1}{(p-k)^{2}+i \varepsilon} \\
& =\int d k^{-} \frac{\tilde{k}^{-}+\left(k^{-}-\tilde{k}^{-}\right)}{\left(k^{2}-m_{q}^{2}+i \varepsilon\right)^{2}\left[(p-k)^{2}+i \varepsilon\right]} \\
& =f_{\text {can. }}\left(k^{+}, \mathbf{k}_{\perp}\right)+f_{\text {sin. }}\left(k^{+}, \mathbf{k}_{\perp}\right),
\end{aligned}
$$

where the 'canonical' piece $f_{\text {can }}$. is obtained by substituting for $k^{-}$its on energy-shell value $\tilde{k}^{-}(2.29)$ (the value at the pole at $(p-k)^{2}=0$ )

$$
\tilde{k}^{-} \equiv p^{-}-\frac{\left(\mathbf{p}_{\perp}-\mathbf{k}_{\perp}\right)^{2}}{2\left(p^{+}-k^{+}\right)} .
$$

For $k^{+}=x p^{+} \neq 0$, it is only this canonical piece which contributes. To see this, we note that

$$
k^{-}-\tilde{k^{-}}=-\frac{(p-k)^{2}}{2\left(p^{+}-k^{+}\right)}
$$

and therefore

$$
\begin{aligned}
f_{\text {sin }}\left(k^{+}, \mathbf{k}_{\perp}\right) & =\int d k^{-} \frac{k^{-}-\tilde{k}^{-}}{\left(k^{2}-m_{q}^{2}+i \varepsilon\right)^{2}} \frac{1}{(p-k)^{2}+i \varepsilon} \\
& =\frac{1}{2\left(p^{+}-k^{+}\right)} \int d k^{-} \frac{1}{\left(k^{2}-m_{q}^{2}+i \varepsilon\right)^{2}}
\end{aligned}
$$

\footnotetext{
${ }^{4}$ This is the highest power of $k^{-}$that can appear in the numerator for twist three distributions.

${ }^{5}$ There is another point, $k^{+}=p^{+}$, where a similar divergence occurs, but the latter is only logarithmic.
} 
Obviously 17]

$$
\int d k^{-} \frac{1}{\left(2 k^{+} k^{-}-\mathbf{k}_{\perp}^{2}-m_{q}^{2}+i \varepsilon\right)^{2}}=0
$$

for $k^{+} \neq 0$ because then one can always avoid enclosing the pole at $k^{-}=\frac{m_{q}^{2}+\mathbf{k}_{\perp}^{2}-i \varepsilon}{2 k^{+}}$by closing the contour in the appropriate half-plane of the complex $k^{-}-$plane. However, on the other hand

$$
\int d k^{+} d k^{-} \frac{1}{\left(2 k^{+} k^{-}-\mathbf{k}_{\perp}^{2}-m_{q}^{2}+i \varepsilon\right)^{2}}=\int d^{2} k_{L} \frac{1}{\left(k_{L}^{2}-\mathbf{k}_{\perp}^{2}-m_{q}^{2}+i \varepsilon\right)^{2}}=\frac{i \pi}{\mathbf{k}_{\perp}^{2}+m_{q}^{2}}
$$

and therefore

$$
f_{\text {sin }}\left(k^{+}, \mathbf{k}_{\perp}\right)=\frac{1}{2 p^{+}} \frac{i \pi \delta\left(k^{+}\right)}{\mathbf{k}_{\perp}^{2}+m_{q}^{2}}
$$

Upon collecting all terms $\propto k^{-}$in the numerator of Eq. (2.26), and applying Eq. (2.40) to those terms we find after some algebra those terms in $h_{L}\left(x, Q^{2}\right)$ that are singular at $x=0$

$$
h_{L, \sin }\left(x, Q^{2}\right)=-\frac{\alpha_{S}}{2 \pi} \ln \frac{Q^{2}}{m_{q}^{2}} C_{F} \delta(x) .
$$

Together with Eq. (2.31), this gives our final result for $h_{L}$, up to $\mathcal{O}\left(\alpha_{S}\right)$, valid also for $x=0$

$$
h_{L}\left(x, Q^{2}\right)=\delta(x-1)+\frac{\alpha_{S}}{2 \pi} C_{F} \ln \frac{Q^{2}}{m_{q}^{2}}\left[-\delta(x)+\frac{2}{[1-x]_{+}}+\frac{1}{2} \delta(x-1)\right] .
$$

As expected, $h_{L}$ from Eq. (2.42) does now satisfy the $h_{L}$-sum rule, provided of course the origin is included in the integration.

This result is important for several reasons. First of all it confirms our result for $h_{L}\left(x, Q^{2}\right)$ as determined from the moment relations. Secondly, it provides us with a method for calculating these $\delta(x)$ terms and thus enabling us to address the issue of validity of the naive sum rules more systematically. And finally, it shows that there is a close relationship between these $\delta(x)$ terms and the infamous zero-modes in LF field theory [18].

While we were completing the manuscript for this paper, we learned of Ref. [15], where canonical Hamiltonian light-cone perturbation theory is used to calculate $h_{L}(x)$. For $x \neq 0$ the result obtained in Ref. [15] agrees with ours which provides an independent check of the formalism and the algebra. However, the canonical light-cone perturbation theory used in Ref. [15] is not adequate for studying the point $x=0$. From the smooth behaviour of $h_{L}(x)$ near $x=0$ the authors of Ref. [15 conclude that the sum rule for the parton distribution $h_{L}(x)$ is violated to $\mathcal{O}\left(\alpha_{S}\right)$. Our explicit calculation for $h_{L}(x)$ not only proves that the sum rule for $h_{L}(x)$ is not violated to this order if the point $x=0$ is properly included, but also shows that it is incorrect to draw conclusions from smooth behaviout near $x=0$ about the behaviour at $x=0$. 


\section{$3 e\left(x, Q^{2}\right)$}

The other chiral-odd twist-3 distribution $e\left(x, Q^{2}\right)$ is also expected to satisfy a simple operator sum rule

$$
\int_{-1}^{1} d x e\left(x, Q^{2}\right)=\frac{1}{2 M}\left\langle P|\bar{\psi}(0) \psi(0)|_{Q^{2}} \mid P\right\rangle
$$

which follows trivially by integrating (1.3) over $x$. Because of our results from above for $h_{L}\left(x, Q^{2}\right)$, we are now of course more cautious and address in the following the issue whether Eq. (3.1) is also valid if the origin is excluded from the region of integration. Again, we will consider a massive quark to $\mathcal{O}\left(\alpha_{S}\right)$

\subsection{Constructing $e\left(x, Q^{2}\right)$ from its moments}

The OPE analysis of (1.3) decomposes $e(x)$ into the twist-2 contribution and the purely twist-3 piece $e^{3}(x)$ as

$$
\mathcal{M}_{n}[e]=\mathcal{M}_{n}\left[e^{3}\right]+\frac{m_{q}}{M} \mathcal{M}_{n-1}\left[f_{1}\right], \quad(n \geq 1)
$$

with the relation (3.1). This moment relation defines the decomposition

$$
x e\left(x, Q^{2}\right)=x e^{3}\left(x, Q^{2}\right)+\frac{m_{q}}{M} f_{1}\left(x, Q^{2}\right) .
$$

where we multiplied $x$ to regularize any possible $\delta(x)$ contributions, which will be specified later. The one loop calculation of $e\left(x, Q^{2}\right)$ for a massive quark yields

$$
e\left(x, Q^{2}\right)=\delta(1-x)+\frac{\alpha_{S}}{2 \pi} C_{F} \ln \frac{Q^{2}}{m_{q}^{2}} e^{(1)}(x) .
$$

The lowest moment of $e^{(1)}(x)$ can be obtained directly from the $\sigma$-term relation (3.1)

$$
\mathcal{M}_{0}[e]=\frac{1}{2 M}\langle P|\bar{\psi} \psi| P\rangle=\frac{1}{2 M} \frac{\partial}{\partial m_{q}} M^{2}=\frac{\partial M}{\partial m_{q}}=1+\frac{\alpha_{S}}{2 \pi} C_{F} \times \frac{3}{2} \ln \frac{Q^{2}}{m_{q}^{2}}
$$

i.e. the lowest moment of $e^{(1)}$ reads

$$
\mathcal{M}_{0}\left[e^{(1)}\right]=\frac{3}{2}
$$

Corresponding to (3.2), we have

$$
\mathcal{M}_{n}\left[e^{(1)}\right]=\mathcal{M}_{n}\left[e^{3(1)}\right]+\frac{1}{M} \mathcal{M}_{n-1}\left[\left(m_{q} f_{1}\right)^{(1)}\right], \quad(n \geq 1)
$$


Our problem is to construct $e^{(1)}(x)$ for a massive quark from (3.6) and (3.7). In principle we can obtain the purely twist-3 piece $e^{3(1)}$ from the one-loop calculation starting from the correlation function [11, 12, 13]:

$$
e^{3}\left(x, Q^{2}\right)=\frac{P^{+}}{M} \int \frac{d \xi^{-}}{2 \pi} e^{2 i x P \cdot \xi} \int_{0}^{1} d u \int_{-u}^{u} d t\left\langle P\left|\bar{\psi}(-u \xi) \sigma^{\mu \alpha} g F_{\nu \alpha}(t \xi) \xi_{\mu} \xi^{\nu} \psi(u \xi)\right|_{Q^{2}} \mid P\right\rangle .
$$

However, we again make a short cut to get it from the moment. The moment of $e^{3(1)}(x)$ for a massive quark is given in [19 as a part of the mixing matrix in the context of the renormalization. From eqs.(4.2) and (3.18) of [19] we have for the $n$-th moment of $e^{3(1)}$ $(n \geq 1)$ :

$$
\begin{aligned}
& \int_{-1}^{1} d x x^{n} e^{3(1)}(x)= \begin{cases}2 \sum_{l=2}^{n / 2}\left[\frac{1}{[l-1]_{3}}+\frac{1}{[n-l+1]_{3}}\right]+\frac{2}{[n / 2]_{3}}, & (n: \text { even }) \\
2 \sum_{l=2}^{(n+1) / 2}\left[\frac{1}{[l-1]_{3}}+\frac{1}{[n-l+1]_{3}}\right], & (n: \text { odd })\end{cases} \\
& =\frac{1}{2}-\frac{1}{n}+\frac{1}{n+1}
\end{aligned}
$$

which gives

$$
x e^{3(1)}(x)=x \frac{1}{2} \delta(1-x)-1+x
$$

Together with the twist-2 contribution

$$
\frac{1}{M}\left(m_{q} f_{1}\right)^{(1)}(x)=\frac{1+x^{2}}{[1-x]_{+}},
$$

one obtains

$$
e^{(1)}(x)=\frac{2}{[1-x]_{+}}+\frac{1}{2} \delta(1-x) \quad \text { for } \quad x>0
$$

Note that $1 / x$ singularity in $e^{3(1)}(x)$ and $f_{1}^{(1)} / x$ cancel each other in $e^{(1)}$, and $e^{(1)}(x)$ itself is integrable at $x=0$. In order to satisfy the $\sigma$-term sum rule (3.6) one needs to introduce $\delta(x)$ term. Accordingly the final result, which satisfies the moment relations both for $n=0$ and $n \neq 0$, reads

$$
e^{(1)}(x)=\frac{2}{[1-x]_{+}}+\frac{1}{2} \delta(1-x)+\delta(x) .
$$

This result clearly indicates that the sum rule (3.1) is satisfied only by including the point $x=0$ in the integration. 


\subsection{Light-cone calculation}

We start from Eq. (2.26) with $\Gamma=1$

$e(x) \bar{u}(p) \Gamma u(p)=-i g^{2} \bar{u}(p) \int \frac{d^{4} k}{(2 \pi)^{4}} \delta\left(x-\frac{k^{+}}{p^{+}}\right) \gamma^{\mu} \frac{1}{\not k-m_{q}+i \varepsilon} \frac{1}{\not k-m_{q}+i \varepsilon} \gamma^{\nu} u(p) D_{\mu \nu}(p-k)$.

the numerator algebra in Eq. (3.14) yields

$$
\begin{aligned}
& \bar{u}(p) \gamma_{\mu}\left(\not k+m_{q}\right)^{2} \gamma_{n} u u(p)\left[g^{\mu \nu}-\frac{(p-k)^{\nu} n^{\mu}+(p-k)^{\mu} n^{\nu}}{p^{+}-k^{+}}\right] \\
= & 2 \bar{u}(p)\left[k^{2}+m_{q}^{2}+2 \frac{k^{2} p^{+}-m_{q}^{2} k^{+}}{p^{+}-k^{+}}-2 m_{q} \not k\right] u(p) .
\end{aligned}
$$

In order to determine the 'canonical' part of $e\left(x, Q^{2}\right)$ we use again contour integration, picking up the pole at $k^{-}=\tilde{k}^{-}$for $0<k^{+}<p^{+}$, which allows us to replace $k^{2} \rightarrow-\frac{\mathbf{k}_{\perp}^{2}}{1-x}$ and $p^{+} k^{-} \rightarrow-\frac{\mathbf{k}_{\perp}^{2}}{2(1-x)}$, where we kept only the leading terms in $\mathbf{k}_{\perp}$. After some algebra this yields for $0<x<1$

$$
e^{(1)}(x)=\frac{2}{1-x}
$$

in agreement with the result obtained from the moments. For the wave function renormalization (the coefficient in front of $\delta(x)$ the same coefficient is obtained as for $h_{L}\left(x, Q^{2}\right)$ and the details will be omitted here.

Finally, we focus on possibly singular terms near $x=0$. The only numerator term involving $k^{-}$in Eq. (3.15), which is not multiplied by $k^{+}$appears in $-4 m_{q} \bar{u}(p) \not k u(p)$. Upon repeating the same steps as in Section 3, i.e. isolating the singular piece by adding and subtracting $\tilde{k}^{-}$, canceling $k^{-}-\tilde{k}^{-}$against the gluon propagator, and making use of Eq. (2.40) one finds

$$
e^{(1)}(x)_{\sin }=\delta(x)
$$

Collecting all terms we thus find

$$
e^{(1)}(x)=\frac{2}{[1-x]_{+}}+\frac{1}{2} \delta(1-x)+\delta(x)
$$

which completely agrees with the result from above (3.13) 


\section{$4 g_{T}\left(x, Q^{2}\right)$}

\subsection{Possibility of $\delta(x)$ in $g_{T}$}

From a practical point of view, $g_{T}\left(x, Q^{2}\right)$ is the most important among the twist-3 distributions, because it is the least difficult to measure experimentally. For this reason, $g_{T}\left(x, Q^{2}\right)$ has been the subject of many studies in the literature.

We again start with the decomposition of $g_{T}(x)$ into the Wandzura-Wilcek term $g_{T}^{W W}(x)$ [20], the term proportional to the quark mass $g_{T}^{m}(x)$, and the purely twist-3 part $g_{T}^{3}(x)$ : o

$$
\begin{aligned}
g_{T}(x)= & g_{T}^{W W}(x)+g_{T}^{m}(x)+g_{T}^{3}(x) \\
& =\left\{\begin{array}{cc}
\int_{x}^{1} d y \frac{g_{1}(y)}{y}+\frac{m_{q}}{M}\left[\frac{h_{1}(x)}{x}-\int_{x}^{1} d y \frac{h_{1}(y)}{y^{2}}\right]+g_{T}^{3}(x) & (x>0), \\
-\int_{-1}^{x} d y \frac{g_{1}(y)}{y}+\frac{m_{q}}{M}\left[\frac{h_{1}(x)}{x}+\int_{-1}^{x} d y \frac{h_{1}(y)}{y^{2}}\right]+g_{T}^{3}(x) & (x<0) .
\end{array}\right.
\end{aligned}
$$

This decomposition is obtained by the analysis of the correlation function using QCD equation of motions. The corresponding moment relations are

$$
\begin{aligned}
& \mathcal{M}_{n}\left[g_{T}\right]=\frac{1}{n+1} \mathcal{M}_{n-1}\left[g_{1}\right]+\frac{n}{n+1} \frac{m_{q}}{M} \mathcal{M}_{n-1}\left[h_{1}\right]+\mathcal{M}_{n}\left[g_{T}^{3}\right], \quad(n \geq 1) \\
& \mathcal{M}_{0}\left[g_{T}\right]=\mathcal{M}_{0}\left[g_{1}\right],
\end{aligned}
$$

with

$$
\mathcal{M}_{0}\left[g_{T}^{3}\right]=0
$$

where we assumed that $g_{T}^{3}(x)$ itself is integrable at $x=0$. The relation (4.4) is known as the Burkhardt-Cottingham (BC) sum rule [2]. We note (4.4) and (4.5) implies

$$
\mathcal{M}_{0}\left[g_{T}^{m}\right]=0 .
$$

In the above discussion we again assumed separate relations (4.5) and (4.6) instead of $\mathcal{M}_{0}\left[g_{T}^{m}+g_{T}^{3}\right]=0$. This is justified as long as $h_{1}(0 \pm)$ is finite. (See below.)

Following the same argument leading to (2.12) for $h_{L}^{m}(x)$, we have

$$
\int_{-1}^{0-} d x g_{T}^{m}(x)+\int_{0+}^{1} d x g_{T}^{m}(x)=\frac{m_{q}}{M}\left(h_{1}(0+)-h_{1}(0-)\right) .
$$

This relation together with (4.6) implies $g_{T}^{m}(x)$ has a singularity at $x=0$ as

$$
g_{T}^{m}(x)=\left.g_{T}^{m}(x)\right|_{r e g}-\frac{m_{q}}{M}\left(h_{1}(0+)-h_{1}(0-)\right) \delta(x),
$$

\footnotetext{
${ }^{6}$ Here and below in this subsection we omit the scale dependence of the distribution functions for simplicity.
} 
where $\left.g_{T}^{m}(x)\right|_{\text {reg }}$ is a part obtained from the integral (4.2) at $x>0$ and $x<0$ and is regular at $x=0$. This relation shows that if $h_{1}(0-) \equiv-\bar{h}_{1}(0+) \neq h_{1}(0+)$ then the BurkhardtCottingham relation would be violated if data is taken only for nonzero $x$ - unless of course there is another $\delta(x)$ contribution to $g_{T}^{3}(x)$ which happens to cancel exactly the one in $g_{T}^{m}$. Perturbation theory predicts at small $x$ that $h_{1}(x) \sim x$ in the leading order as is seen from (2.17) and $h_{1}(x)-h_{1}(-x) \sim$ const. at the next-to-leading order [21]. BFKL approach gives $h_{1}(x) \sim$ const. at small $x[22]$. Therefore there is a possibility that the $\delta(x)$ term in (4.8) survives and hence a seeming violation of BC sum rule.

In the next subsections, we will calculate $g_{T}\left(x, Q^{2}\right)$ for a massive quark to $\mathcal{O}\left(\alpha_{S}\right)$ in order to look for (possibly) another origin of the $\delta(x)$ contribution.

\subsection{Constructing $g_{T}\left(x, Q^{2}\right)$ from its moments to $\mathcal{O}\left(\alpha_{S}\right)$}

One loop calculation of $g_{T}(x)$ for a massive quark takes the form of

$$
g_{T}\left(x, Q^{2}\right)=\delta(1-x)+\frac{\alpha_{S}}{2 \pi} C_{F} \ln \frac{Q^{2}}{m_{q}^{2}} g_{T}^{(1)}(x) .
$$

Corresponding to the decomposition (4.1) we write

$$
g_{T}^{(1)}(x)=g_{T}^{W W(1)}(x)+g_{T}^{m(1)}(x)+g_{T}^{3(1)}(x) .
$$

Inserting the expression (2.16) for $g_{1}^{(1)}$ and (2.17) for $h_{1}^{(1)}$ into (4.2), and also taking into account the self-energy correction, we have

$$
g_{T}^{W W(1)}(x)+g_{T}^{m(1)}(x)=\ln x+\frac{1}{2}+x+\frac{2}{[1-x]_{+}} .
$$

This result has no $\delta(x)$ term, which is simply because $h_{1}\left(0^{+}\right)=h_{1}\left(0^{-}\right)=0$ in the one-loop calculation. (See (4.8).)

As in the case of $h_{L}^{3}(x)$ and $e^{3}(x)$, we construct $g_{T}^{3(1)}$ from its moment for a massive quark. From eqs. (5) and (18) of [23], we have for the $n$-th moment of $g_{T}^{3(1)}(x)$ as

$$
\begin{aligned}
\int_{-1}^{1} d x x^{n} g_{T}^{3(1)}(x) & =\sum_{l=1}^{n-2}(n-l) \frac{2}{(n+1) l(l+1)(l+2)} \\
& =\frac{1}{2}-\frac{3}{2(n+1)}+\frac{1}{(n+1)^{2}} .
\end{aligned}
$$

This moment relation is originally given for $n \geq 1$, but it also gives zero for $n=0$, which is consistent with (4.5). Accordingly no $\delta(x)$ term is necessary to obtain

$$
g_{T}^{3(1)}(x)=\frac{1}{2} \delta(1-x)-\frac{3}{2}-\ln x
$$

From 4.11) and (4.13), we get

$$
g_{T}\left(x, Q^{2}\right)=\delta(1-x)+\frac{\alpha_{S}}{2 \pi} C_{F} \ln \frac{Q^{2}}{m_{q}^{2}}\left[\frac{1+2 x-x^{2}}{[1-x]_{+}}+\frac{1}{2} \delta(1-x)\right] .
$$




\subsection{LC calculation}

We calculated $g_{T}\left(x, Q^{2}\right)$, using using the same LF pQCD techniques that we used to study $h_{L}\left(x, Q^{2}\right)$ and $e\left(x, Q^{2}\right)$. The algebraic steps involved are rather lengthy and we therefore present only the final result here, which reads

$$
g_{T}\left(x, Q^{2}\right)=\delta(1-x)+\frac{\alpha_{S}}{2 \pi} C_{F} \ln \frac{Q^{2}}{m_{q}^{2}}\left[2 x+\frac{1+x^{2}}{[1-x]_{+}}+\frac{1}{2} \delta(1-x)\right]
$$

without any $\delta(x)$ term. This result completely agrees with the findings from Ref. [16, which confirms our formalism.

Even though the numerator for $\Gamma=\gamma_{\perp} \gamma_{5}$ in Eq. (2.26) contains $k^{-}$, those terms turn out to be multiplied by at least one power of $k^{+}$. Since $x \delta(x)=0$, there are no $\delta(x)$ terms in $g_{T}\left(x, Q^{2}\right)$ to $\mathcal{O}\left(\alpha_{S}\right)$. However, we do not have a simple explanation (other than working out the numerator algebra) as to why factors of $k^{-}$in the numerator algebra are always accompanied by at least one power of $k^{+}$for $g_{T}\left(x, Q^{2}\right)$ and not for $h_{L}\left(x, Q^{2}\right)$ and $e\left(x, Q^{2}\right)$ in this one loop calculation.

\section{Summary}

We have investigated sum-rules for twist-3 distributions in QCD, and found examples where these sum-rules are violated if the point $x=0$ is not properly included.

For a massive quark, to $\mathcal{O}\left(\alpha_{S}\right)$ we found

$$
\begin{aligned}
g_{T}\left(x, Q^{2}\right) & =\delta(x-1)+\frac{\alpha_{S}}{2 \pi} C_{F} \ln \frac{Q^{2}}{m_{q}^{2}}\left[2 x+\frac{1+x^{2}}{[1-x]_{+}}+\frac{1}{2} \delta(x-1)\right] \\
h_{L}\left(x, Q^{2}\right) & =\delta(x-1)+\frac{\alpha_{S}}{2 \pi} C_{F} \ln \frac{Q^{2}}{m_{q}^{2}}\left[-\delta(x)+\frac{2}{[1-x]_{+}}+\frac{1}{2} \delta(x-1)\right] \\
e\left(x, Q^{2}\right) & =\delta(x-1)+\frac{\alpha_{S}}{2 \pi} C_{F} \ln \frac{Q^{2}}{m_{q}^{2}}\left[\delta(x)+\frac{2}{[1-x]_{+}}+\frac{1}{2} \delta(x-1)\right] .
\end{aligned}
$$

At $\mathcal{O}\left(\alpha_{S}\right)$ neither $h_{L}\left(x, Q^{2}\right)$ nor $e\left(x, Q^{2}\right)$ satisfy their respective sum rule if one excludes the origin from the region of integration (which normally happens in experimental attempts to verify a sum rule). $g_{T}\left(x, Q^{2}\right)$ is the only exception and its sum-rule is satisfied even when the origin is not included.

Of course, QCD is a strongly interacting theory and parton distribution functions in QCD are nonperturbative observables. Nevertheless, if one can show that a sum rule fails already in perturbation theory, then this is usually a very strong indication that the sum rule also fails nonperturbatively (while the converse is often not the case!). 
From the QCD equations of motion, we were able to show nonperturbatively that $\overline{1}$ the difference between $h_{L}\left(x, Q^{2}\right)$ and $h_{L}^{3}\left(x, Q^{2}\right)$ contains a $\delta$ function at $x=0$

$$
\left[h_{L}\left(x, Q^{2}\right)-h_{L}^{3}\left(x, Q^{2}\right)\right]_{\text {singular }}=-\frac{m_{q}}{2 M}\left(g_{1}\left(0+, Q^{2}\right)-g_{1}\left(0-, Q^{2}\right)\right) \delta(x) .
$$

Since $g_{1}\left(0+, Q^{2}\right)-g_{1}\left(0-, Q^{2}\right) \equiv \lim _{x \rightarrow 0} g_{1}\left(x, Q^{2}\right)-\bar{g}_{1}\left(x \cdot Q^{2}\right)$ seems to be nonzero (it may even diverge $\left.{ }^{\mathbb{P}}\right)$, one can thus conclude that either $h_{L}\left(x, Q^{2}\right)$ or $h_{L}^{3}\left(x, Q^{2}\right)$ or both do contain such a singular term.

We checked the validity of this relation to $\mathcal{O}\left(\alpha_{S}\right)$ and found that, to this order, both $h_{L}^{3}$ and $h_{L}$ contain a term $\propto \delta(x)$. We also verified that even though the sum rule for $h_{L}(x)$ and $e(x)$ are violated if $x=0$ is not included, the sum rules for all three twist 3 parton distributions are still satisfied to $\mathcal{O}\left(\alpha_{S}\right)$ if the contribution from $x=0$ (the $\delta(x)$ term) is included.

\section{Acknowledgments}

M.B. was supported by a grant from the DOE (FG03-95ER40965), through Jefferson Lab by contract DE-AC05-84ER40150 under which the Southeastern Universities Research Association (SURA) operates the Thomas Jefferson National Accelerator Facility. Y.K. is supported by the Grant-in-Aid for Scientific Research (No. 12640260) of the Ministry of Education, Culture, Sports, Science and Technology (Japan). We are also greatful to JSPS for the Invitation Fellowship for Research in Japan (S-00209) which made it possible to materialize this work.

\section{References}

[1] R.L. Jaffe and X. Ji, Nucl. Phys. B375 (1992) 527.

[2] H. Burkhardt and W.N. Cottingham, Ann. of Phys. 56 (1970) 453.

[3] M. Burkardt, Proc. to Spin 92, Eds. Hasegawa et al., (Universal Academy Press, Tokyo, 1993).

[4] R.D. Tangerman and P.J. Mulders, hep-ph/0408305.

[5] M. Burkardt, Phys. Rev. D52 (1995) 3841.

[6] R. Jakob, P.J. Mulders, J. Rodrigues,Nucl. Phys. A 626 (1997) 937.

[7] D. Boer, Ph.D. Thesis, Vrije U., Amsterdam, 1998.

\footnotetext{
${ }^{7}$ The only assumption that we made is that twist- 2 distributions do not contain a $\delta$-function at $x=0$.

${ }^{8}$ In the next-to-leading order QCD for a quark, $\lim _{x \rightarrow 0} g_{1}(x)-\bar{g}_{1}(x)$ is logarithmically divergent [24].
} 
[8] M. Burkardt, in "Exclusive and semi-exclusive processes at high momentum transfer", Eds. C. Carlson and A. Radyushkin, Newport News, VA, May 1999; hep-ph/9908479.

[9] V.N. Gribov and L.N. Lipatov, Sov. J. Nucl. Phys. 15 (1972) 438; Yu. L. Dokshitser, Sov. Phys. JETP, 46 (1977) 641; G. Altarelli and G. Parisi, Nucl. Phys. B126 (1977) 298.

[10] X. Artru and M. Mekhfi, Z. Phys. C 45 (1990) 669.

[11] I.I. Balitsky, V.M. Braun, Y. Koike and K. Tanaka, Phys. Rev. Lett. 77 (1996) 3078.

[12] A.V. Belitsky and D.Müller, Nucl. Phys. B503 (1997) 279.

[13] J. Kodaira and K. Tanaka, Prog. Theor. Phys. 101 (1999) 191.

[14] Y. Koike and K. Tanaka, Phys. Rev. D 51 (1995) 6125.

[15] R. Kundu and A. Metz, hep-ph/0107073.

[16] A. Harindranath and W.-M. Zhang, Phys. Lett. B 408 (1997) 347.

[17] S.-J. Chang and T.-M. Yan, Phys. Rev. D 7 (1972) 1147.

[18] M. Burkardt, Advances Nucl. Phys. 23 (1996) 1.

[19] Y. Koike and N. Nishiyama, Phys. Rev. D 55 (1997) 3068.

[20] S. Wandzura and F. Wilczek, Phys. Lett. B 72 (1977) 195.

[21] A. Hayashigaki, Y. Kanazawa and Y. Koike, Phys. Rev. D 56 (1997) 7350;

W. Vogelsang, Phys. Rev. D 57 (1998) 1886.

[22] R. Kirschner, L. Mankiewicz, A. Schäfer and L. Szymanowski, Z. Phys. C74 (1997) 501.

[23] J. Kodaira, Y. Yasui, K. Tanaka and T. Uematsu, Phys. Lett. B 387 (1996) 855.

[24] W. Vogelsang, Phys. Rev. D54 (1996) 2023; Nucl. Phys. B457 (1996) 47; R. Mertig and W.L. van Neerven, Z. Phys. C70 (1996) 637. 